\title{
La especificidad de la enseñanza-aprendizaje de la lengua extranjera en la carrera de Tra- ducción
}

\author{
Astrid Schmidhofer \\ Universidad Europea de Madrid
}

https://dx.doi.org/10.12795/futhark.2014.i9.16

\begin{abstract}
The purpose of this article is to outline the bases of a specific methodology for the teaching of foreign languages within Translation degree programmes. As a starting point we will define specific learning goals and compare them with those established for communicative language learning. We will then examine up to which extent the communicative methodology is applicable and appropriate for the learning context at hand. Based on the specific learning goals, we will outline a methodology that combines communicative elements, grammatical accuracy and contrastive teaching.
\end{abstract}

Keywords: second language learning, translation, contrastive teaching

Resumen: El objetivo de este artículo es proponer una metodología específica para la enseñanza de la segunda lengua extranjera en las carreras de Traducción. Como punto de partida se describen el contexto educativo y los objetivos específicos de esta enseñanza, comparándolos con los objetivos de una enseñanza de idiomas comunicativa con el fin de valorar hasta qué punto la metodología comunicativa, que es la más extendida en Europa hoy día, es eficaz y adecuada en este contexto educativo. Tras la definición de dichos objetivos, se trazarán las bases de una metodología específica que combina elementos comunicativos, precisión gramatical y enseñanza contrastiva. 
Palabras clave: Enseñanza de segundas lenguas, traducción, ensefianza contrastiva, carrera de Traducción

\section{INTRODUCCIÓN}

En las carreras de Traducción en España, durante los primeros dos cursos, se dedica una parte considerable del tiempo a la adquisicionaprendizaje de las lenguas extranjeras que serán, aparte de la lengua materna, las herramientas de trabajo en las asignaturas de Traduccion. La enseñanza de estas lenguas debe enfocarse, por lo tanto, al aprendizaje de las habilidades necesarias para poder afrontar la futura labor traductora. En este artículo definiremos detalladamente los objetivos de esta enseñanza específica de lenguas y los compararemos con los objetivos que se persiguen en una enseñanza con fines comunicativos. Basándonos en los objetivos establecidos, esbozaremos unas bases metodológicas que, a nuestro parecer, son las más apropiadas para la enseñanza de idiomas en el contexto de las carreras de Traducción y valoraremos en qué medida deben integrarse elementos propios del enfoque comunicativo.

La enseñanza-aprendizaje de segundas lenguas cuenta con una larga tradición que se ve reflejada en la amplísima bibliografía que existe en este ámbito. Asimismo, las últimas dos décadas han visto un aumento significativo de las monografías y artículos dedicados a diversos aspectos de la Didáctica de la Traducción. ${ }^{1}$ Sin embargo, existe relativamente poca bibliografía sobre el campo limítrofe entre estas dos áreas que aquí nos ocupa. Las publicaciones del ámbito español comprenden los artículos de Laura Berenguer (1996 y 1999), quien formula cinco objetivos clave para la Enseñanza de Lenguas a traductores y aporta además una serie de ejercicios prácticos para el aula, de Pilar Civera (1996), quien desarrolla el trabajo de las cuatro destrezas,

\footnotetext{
${ }^{1}$ En el ámbito español cabe destacar las colecciones dirigidas por Amparo Hurtado Albir La enseñanza de la Traducción (1996) y Enseñar a traducir (1999) que recogen artículos de distintos autores acerca de diferentes aspectos de la Enseñanza de la
Traducción.
} 
de Justine Brehm Cripps y Amparo Hurtado Albir (1999) y Pilar Civera, Ulrike Oster y Amparo Hurtado Albir (1999), quienes presentan objetivos y actividades, de Carmen Gómez García (2002-2003), quien incide en la importancia del papel de la gramática, de María del Carmen Martín Santana (2004), quien propone integrar elementos del método de gramática-traducción en la enseñanza de lenguas a traductores, y de María Ángeles Recio Ariza, quien resalta el papel de la gramática y de la cultura en la enseñanza de la Lengua $C$. A todas ellas nos referiremos en las disquisiciones presentadas en las siguientes páginas.

En este artículo, nos centraremos específicamente en la enseñanzaaprendizaje de la lengua $C$, que suele impartirse desde un nivel inicial en la mayoría de las carreras de Traducción que se ofrecen en el ámbito español.

\section{EL CONTEXTO DE ENSEÑANZA DE LA LENGUA C EN LAS CARRERAS DE TRADUCCIÓN}

La mayoría de los estudiantes que inician sus estudios de Traducción eligen como primera lengua extranjera el inglés, pues para la primera lengua extranjera (lengua B) suele establecerse un nivel mínimo como requisito de admisión (conocimientos entre los niveles B1 y el C1 según el MCER) y muchos estudiantes solo cumplen dicho requisito en la lengua inglesa. Por lo tanto, la enseñanza de la lengua C suele enfocarse a idiomas distintos del inglés, en la mayoría de los casos el alemán y el francés. La oferta concreta depende, sin embargo, de cada centro. ${ }^{2}$

Puesto que en la lengua $C$ no se exigen conocimientos previos y la enseñanza-aprendizaje comienza, por lo tanto, en el nivel A1, se diferencia de la enseñanza de la lengua $B$ en los siguientes aspectos (cf. también los comentarios de Recio Ariza 2008: 279 al respecto):

${ }^{2}$ En el caso de nuestra universidad (la Universidad Europea de Madrid) se ofrece alemán, francés y chino. 
Los estudiantes ya poseen conocimientos previos de la lengua B (un gran número de ellos lleva muchos años estudiando dicha lengua o bien en la enseñanza reglada y/o en academias privadas o en escuelas de verano en el extranjero), por lo que están acostumbrados a usarla tanto en el contexto educativo como en su vida personal y están familiarizados en mayor o menor medida con la cultura de los países donde se habla dicho idioma. En pocas palabras, la lengua $B$ ya forma parte de su vida y de su inventario lingüístico, lo que, desde el punto de la adquisición y la integración de nuevos conocimientos, es un aspecto determinante. En consecuencia, los objetivos de la enseñanza de la lengua $B$ deben establecerse conforme a esta situación de partida y regirse por una progresion acorde con estas premisas (cf. los objetivos y la progresión expuestos en Brehm Cripps y Hurtado Albir 1999: 59-65).

En cambio, en el caso de la lengua $C$, la situación de partida es muy diferente, puesto que, al menos una parte de los estudiantes, no suele poseer conocimientos previos. Esto implica que además de los conocimientos lingüísticos, los estudiantes deben adquirir todo el bagaje cultural necesario para su futura labor traductora. Asimismo, deben integrar el idioma nuevo en su inventario lingüistico y adquirir el hábito de utilizarlo como herramienta de comunicación e interacción. Para tal fin, es necesario promover todo tipo de actividades que suponen el uso del idioma (con uso nos referimos tanto a la recepción como la producción) en contextos auténticos, es decir, actividades conjuntas con hablantes nativos, visitas culturales, lecturas, visionados de películas o el uso de Internet y de las redes sociales. Estas actividades deben ser complementarias a la enseñanza de la lengua en las asignaturas de Lengua $C$, lo que supone una inversión de tiempo considerable por parte del alumnado y del profesorado. Sin embargo, solo a través de un esfuerzo personal considerable, junto a una enseñanza específica, podrán adquirir los estudiantes, en un espacio de dos años, el dominio suficiente para poder enfrentarse a las asignaturas de Traducción en las que deberán trabajar con textos auténticos de diferentes campos temáticos. 
3. LOS OBJETIVOS DE LA ENSEÑANZA-APRENDIZAJE DE IDIOMAS EN LAS CARRERAS DE TRADUCCIÓN EN COMPARACIÓN CON LOS OBJETIVOS DE LA ENSEÑANZAAPRENDIZAJE DE IDIOMAS CON FINES COMUNICATIVOS

\subsection{MOTIVACIÓN Y OBJETIVOS}

En este apartado analizaremos hasta qué punto existe una coincidencia entre la enseñanza-aprendizaje de lenguas en las carreras de Traducción con aquella enfocada a fines puramente comunicativos. Como punto de partida estableceremos los objetivos y motivaciones de los dos contextos elegidos.

La enseñanza de idiomas con fines comunicativos se lleva a cabo en entornos educativos muy diversos, tanto institucionalizados (asignaturas de idiomas en carreras universitarias con orientación internacional, Escuelas Oficiales de Idiomas, programas de formación para desempleados o formación continua para trabajadores) como de enseñanza no reglada (centros de estudios, academias, centros culturales, empresas, etc.). Las motivaciones también suelen ser diversas y pueden agruparse en personales (deseo de comunicarse con nativos de la lengua meta por amistad, durante un viaje, para integrarse en una sociedad de la lengua meta o por interés en la cultura meta), laborales (para cumplir con las exigencias de un determinado puesto de trabajo o las exigencias del mercado laboral) o educativas (para superar un programa formativo) o una combinación de varias de estas motivaciones.

El objetivo global común suele consistir en alcanzar una comunicación eficaz y apropiada para diferentes contextos sociales ${ }^{3}$. La gran

${ }^{3}$ Este objetivo se encuentra reflejado en muchos de libros de texto actualmente en uso. Citaremos como ejemplos del ámbito del alemán los libros de texto Studio d (editorial Cornelsen), Menschen (editorial Hueber), Schritte (editorial Hueber) o Netzwerk (editorial Langenscheidt).

Futhark 9 (2014)

Schmidhofer, La especificidad, 425-452

ISSN 1886-9300 

mayoría de los aprendientes formulan, además, los siguientes objeti.
vos ${ }^{4}$ específicos:

- Expresarse con soltura en la lengua meta, especialmente en la comunicación oral

- Comprender a interlocutores nativos y no-nativos

- Interactuar con hablantes de otras lenguas de forma fluida en situaciones cotidianas

- Alcanzar un dominio fonético aceptable

- Conocer el léxico asociado a las situaciones comunicativas más frecuentes

- Familiarizarse con los fenómenos culturales de la lengua meta asociados a la interacción comunicativa cotidiana

Asimismo, este aprendiente muestra las siguientes actitudes:

- Interés destacado por la práctica oral

- Preferencia por los temas cotidianos

- Interés muy dispar con respecto a la precisión

- Interés somero por los fenómenos culturales de la lengua meta

- Tiempo limitado que puede dedicar al estudio

En cambio, en la enseñanza de idiomas en los programas de Traducción, las motivaciones son, principalmente, educativas, pues los estudiantes cursan estas asignaturas como parte de un programa universitario al final del cual obtendrán un título que les facilitará el acceso al mercado laboral. Por lo tanto, los objetivos de esta enseñanzaaprendizaje deben definirse principalmente en función de las competencias que se necesitan para poder afrontar las posteriores asignaturas de Traducción y las exigencias del mercado laboral. De ahí que se

\footnotetext{
${ }^{4}$ Recogidos durante nuestra experiencia personal de más de doce años enseñando alemán en España en diferentes contextos de enseñanza no reglada (academias, clases particulares, clases en empresas y ministerios).
} 
establezcan los siguientes objetivos enfocados a la futura labor traductoras:

- Conocer en profundidad las estructuras de la lengua meta

- Ampliar los conocimientos léxicos, especialmente los receptivos

- Desarrollar a fondo la comprensión lectora tanto global como detallada

- Desarrollar la capacidad para detectar las diferencias estructurales entre la lengua meta y la lengua materna

- Conocer las convenciones de escritura tanto a nivel estilístico como textual de la lengua meta

- Conocer los mecanismos de coherencia y cohesión textuales

- Adquirir un cierto bagaje cultural de la cultura meta

5 Hemos tomado como referencia los objetivos formulados por Laura Berenguer (1996:14) que se pueden resumir de la siguiente manera:

1) Desarrollar la comprensión lectora

2) Aprender a disociar las dos lenguas en contacto

3) Preparar al alumno para la utilización de diccionarios y otras obras de consulta

4) Hacer del futuro traductor un experto en cultura

5) Sensibilizar al alumno a la actividad traductora, tematizando en clase aspectos relacionados con la traducción

María Ángeles Recio Ariza (2008:280) establece la siguiente jerarquía de objetivos para la enseñanza de lenguas a futuros traductores:

1) La enseñanza de la gramática

2) La enseñanza de la cultura

3) El análisis textual

Esta autora también señala que, gracias a los conocimientos previos, la enseñanza de la lengua $B$ puede centrarse más en aspectos estilísticos, mientras que la de la lengua $C$ debe centrarse en cuestiones gramaticales y culturales. 
- Saber utilizar obras de consulta para solucionar cuestiones gramaticales, léxicas, culturales y temáticas

Estos objetivos pueden incluir el desarrollo de la expresión escrita si el plan de estudios incluye una asignatura de traducción inversa en cuyo caso los objetivos formulados para el plano receptivo deben aplicarse también al plano productivo. Asimismo, deben incluir la adquisición de un nivel intermedio en las destrezas orales tanto para cubrir posibles necesidades laborales (los cometidos laborales de un traductor pueden abarcar la interacción con nativos de la lengua meta) $\mathrm{y}$ atender a motivaciones personales, pues muchos estudiantes eligen un idioma determinado por tener contacto con nativos o por interés en la cultura meta. La enseñanza-aprendizaje de las destrezas orales contribuye además a mantener la motivación entre el alumnado y a hacer las clases más interactivas.

La consecución de dichos objetivos depende en gran parte de determinadas actitudes que deben mostrar los estudiantes de Traducción. Estas son, entre otras:

- Interés profundo por la gramática y la precisión

- Interés por fenómenos lingüísticos

- Actitud reflexiva hacia el lenguaje

- Disposición a progresar rápidamente

- Disposición a realizar una importante inversión de tiempo

\section{METODOLOGÍA}

A lo largo de la historia de la enseñanza-aprendizaje de idiomas han surgido una serie de métodos que intentaban proponer soluciones enfocadas a las necesidades sociales relacionadas con el conocimiento de idiomas de cada época. Tómese, como ejemplo, el método de gramática-traducción que permitía adquirir los conocimientos necesarios para leer obras literarias en lengua extranjera o el método comunicativo que surgió en un contexto de intensificación de las relaciones internacionales en diferentes ámbitos. 
Este último método o métodos basados en principios afines como el enfoque por tareas o el método intercultural son los más utilizados hoy día en la enseñanza de idiomas en el ámbito europeo, puesto que son los que mejor se ajustan a los objetivos comunicativos que se juzgan prioritarios en un mundo cada vez más globalizado.

Sin embargo, tal como hemos expuesto en el apartado anterior, existen divergencias importantes entre los objetivos de la enseñanza comunicativa y los objetivos establecidos para la enseñanza de lenguas a traductores. Resulta lógico, por lo tanto, que las metodologías comunicativas no sean del todo adecuadas para la consecución de los objetivos establecidos en el marco de las carreras de Traducción. Para responder a las necesidades específicas de este contexto será necesario, pues, adaptar y complementar las metodologías comunicativas o iniciar el desarrollo de una metodología nueva. ${ }^{6}$ En los siguientes apartados vamos a desarrollar y comentar aquellos aspectos que, en nuestra opinión, podrían formar los pilares básicos para una enseñanza de lenguas en el marco de los programas de Traducción.

\subsection{LAS CUATRO DESTREZAS}

Dado que en las asignaturas de Traducción el trabajo en la lengua extranjera se centra principalmente en la comprensión de textos escritos, el desarrollo de esta destreza debe desempeñar un papel principal en la enseñanza de lenguas a futuros traductores.

Tomando como punto de partida los objetivos establecidos por Pilar Civera (1996:185) ${ }^{7}$ y Pilar Civera, Ulrike Oster y Amparo Hurtado

${ }^{6}$ En los últimos años se han desarrollado y publicado dos libros de texto enfocados específicamente a la enseñanza del alemán a traductores. Son Lengua alemana para traductores coordinado por Laura Berenguer (2006) y Deutsch als Fremdsprache für Übersetzer de Carmen Balbuena Torezano (2007). Desafortunadamente no hemos tenido acceso a estas dos publicaciones.

${ }^{7}$ Estos objetivos son los siguientes:

1) Desarrollo de la comprensión de textos escritos en la lengua $C$, desde el punto de vista de una perspectiva pragmática del funcionamiento de textos: función, destinatario, soporte... 
Albir (1999:72-73) ${ }^{8}$, hemos confeccionado la siguiente relación de objetivos para esta destreza:

- Desarrollar estrategias para la correcta descodificación lingüística de textos escritos en lengua meta ${ }^{9}$

- Aprender a utilizar recursos para lograr dicha descodificación (gramáticas, diccionarios, fuentes documentales, etc.)

- Detectar los mecanismos de coherencia y cohesión presentes en los textos

- Analizar la situación comunicativa que rodea un texto (emisor, texto, destinatario, etc.) y los actos de habla presentes en él

- Conocer las convenciones textuales y estilísticas de la lengua/cultura meta para las diversas situaciones comunicativas

2) Desarrollo de la capacidad de observación de los fenómenos lingüísticos relacionados con la coherencia y la cohesión en la lengua $C$.

3) Desarrollo de la capacidad de observación del funcionamiento textual: descripción de los distintos tipos de texto, de las intenciones de comunicación...

4) Potenciación de su capacidad metacognitiva: evaluación del trabajo personal teniendo en cuenta los objetivos planteados y los resultados obtenidos, con vistas a favorecer el desarrollo de una capacidad autónoma de lectura y de aprendizaje en general.

${ }^{8}$ Estos objetivos son los siguientes:

1) Comprender y asimilar convenciones básicas de la escritura

2) Adquirir estrategias de lectura

3) Identificar y comprender el funcionamiento de los mecanismos de coherencia y de cohesión

4) Identificar y comprender el funcionamiento de distintos tipos y géneros textuales

5) Identificar los rasgos pragmáticos y semióticos de un texto

6) Identificar variaciones lingüísticas relacionadas con el uso

7) Desarrollar un espíritu crítico ante los textos

${ }^{9}$ Como hemos demostrado en un estudio anterior (Astrid Schmidhofer: 2012), la descodificación lingüística sigue planteando problemas en los cursos de Traducción. 
- Familiarizarse con las variantes diatópicas, diastráticas y diafásicas de la lengua meta

- Desarrollar interés por la lectura en lengua meta y hábitos de lectura autónomos

Como puede apreciarse, los primeros tres objetivos se centran en la dimensión lingüística de los textos, los siguientes tres en la dimensión pragmática y el último en la actitud del alumnado.

Para la consecución de los objetivos lingüísticos, proponemos trabajar tanto con textos creados con fines educativos (como, por ejemplo, aquellos incluidos en libros de texto) como textos auténticos. A pesar de las críticas que reciben los primeros por su (supuesta) artificialidad, nos parecen útiles desde un punto de vista didáctico, pues no solo nos permiten centrarnos en fenómenos específicos y ejercitar su comprensión sino también minimizan el peligro de que el alumno se sienta abrumado por un elevado número de estructuras y palabras desconocidas como ocurre a menudo en el trabajo con textos extraídos de fuentes auténticas. Sin embargo, el profesor debe tener siempre presente que los textos creados con fines educativos no hacen sino allanar el camino hacia el trabajo con textos auténticos y nunca son un fin en sí mismo.

El trabajo con textos debe, en nuestra opinión, enfocarse desde dos perspectivas diferentes. Por un lado es necesario desarrollar estrategias de lectura para la comprensión global y detallada. Aquí debe hacerse especial hincapié en los procesos de top-down (activación de conocimientos previos extraídos del propio texto o sobre el mundo) y bottom-up (construcción del sentido a partir de las unidades del texto), pues como demuestra Paul Kussmaul (1995), un desequilibrio en la aplicación de dichos procesos puede acarrear importantes errores de comprensión $^{10}$. Para practicar dichas estrategias, recomendamos los ejercicios que se emplean en el método comunicativo como la formulación de hipótesis sobre el contenido de un texto o una parte del texto $\mathrm{y}$ actividades de diferente índole sobre el contenido global o aspectos

10 Paul Kussmaul (1995: 25-28, 34-37) cita varios ejemplos de Thinking-AloudProtocols que demuestran que los conocimientos sobre el mundo o la experiencia personal pueden inducir a los estudiantes a abusar de los procesos de top-down. 
concretos del texto (existe una gran variedad de tipos de ejercicios como, por ejemplo, preguntas de opción múltiple, ejercicios de enlazar frases incompletas, rellenar huecos, resumir partes del texto u ordenar frases para crear un resumen).

Por otro lado también se debe acostumbrar al alumno a leer los textos desde el punto de vista del traductor, que luego creará un texto de llegada en su lengua materna a partir del texto leído en la lengua extranjera 11 . Para entrenar esta capacidad de transferencia, recomendamos realizar resúmenes en español a partir de textos en la lengua extranjera y traducciones conjuntas en clase que se basen en un análisis sintáctico de las unidades del texto en lengua extranjera. Como estos ejercicios están pensados para entrenar la primera fase del proceso de traducción, recomendamos que no se invierta demasiado tiempo en pulir el texto en lengua meta, pues no es el objetivo de las clases de lengua y puede distraer a los alumnos del trabajo con el texto base que están realizando.

El trabajo con textos originales también debe abarcar el manejo de obras de consulta para solucionar cuestiones léxicas y gramaticales. Esta labor puede realizarse tanto con ejercicios específicos ${ }^{12}$, como mediante el trabajo con los textos en clase. Para el segundo caso, debe elaborarse primero un inventario de fuentes en papel y online ${ }^{13}$ que después se utilizarán para consultar las dudas que vayan surgiendo durante el trabajo con textos. De esta manera, los alumnos comprobarán de primera mano la utilidad de las diferentes obras.

La consecución de los objetivos pragmáticos debe realizarse de forma paralela con el trabajo de descodificación lingüística, puesto que ambos aspectos están íntimamente relacionados: la información pragmática apoya la descodificación lingüística a través de los conocimientos extralingüísticos que los alumnos aportan (top-down) y la

\footnotetext{
11 En este aspecto, la metodología propuesta discrepa de la metodología
comunicativa.

y Amparo Hurtado Albir los ejercicios propuestos por Pilar Civera, Ulrike Oster ${ }_{3}^{3}$ Se petica del manejo de obras de consulta.
dir a los alumnos que confecciones parecen muy eficaces para la

disponibles en la biblioteca del centro y en Internet.
} 
información lingüística ayudará a entender mejor la situación comunicativa (bottom-up). Para facilitar la comprensión de la situación comunicativa que rodea un texto, recomendamos la utilización de una ficha (que los alumnos deberán completar) con información sobre el emisor, el destinatario, el medio, el lugar, el momento, el tipo de texto, las intenciones, los actos de habla y la organización textual. ${ }^{4}$ Esta información también servirá como punto de partida para el análisis de las variantes lingüísticas que aparecen en el texto y que debe centrarse tanto en el nivel léxico como en el sintáctico. Las variantes diafásicas detectadas nos llevan al aspecto de las convenciones de escritura y de organización textual de diferentes tipos de textos, que puede variar considerablemente entre la cultura de origen y meta. Estas diferencias, que son fundamentales para la labor traductora, deben trabajarse desde la contrastividad, es decir, mediante una comparación de un texto en lengua extranjera ya analizado a nivel lingüístico y pragmático con un texto español del mismo tipo.

El último objetivo expuesto en el ámbito de la comprensión escrita abarca el interés por la lectura en lengua extranjera. Los beneficios para la adquisición de léxico y de estructuras a través de la lectura individual son obvios y nos parece innecesario ahondar en ellos. Sí nos gustaría reflexionar sobre los materiales más apropiados para estimular dicho interés. En nuestra experiencia, el uso de lecturas graduadas ha resultado ser una herramienta eficaz para promover la lectura en lengua meta ya en fases iniciales del aprendizaje. A pesar de los inconvenientes conocidos que presentan dichas lecturas (simplificación y trivialización de contenidos, lenguaje poco auténtico), tienen la gran ventaja de que el alumno puede enfrentarse a un texto largo sin hacer un uso excesivo del diccionario, lo que redunda positivamente en la motivación y la actitud hacia la lengua. En cambio, el trabajo con textos que lingüísticamente están muy por encima del nivel de los alumnos conduce a menudo a la frustración y al abandono. Asimismo, dichas lecturas pueden integrarse en las tareas de aprendizaje autónomo, pues se prestan a la elaboración de textos en lengua materna o lengua meta.

${ }^{14}$ Un ejemplo de ficha se encuentra en Laura Berenguer (1996: 21-23). 
Siguiendo lo expuesto en el apartado acerca de los objetivos, la ad. quisición de las otras destrezas dependerá de las asignaturas de Traducción que los alumnos tengan que cursar en la segunda lengua extranjera. Si estas no comprenden asignaturas obligatorias de traducción inversa ni de interpretación de lengua $C$, como es el caso en la mayoría de las universidades españolas ${ }^{15}$, el aprendizaje de las demás destrezas debe efectuarse acorde con las necesidades comunicativas que puedan surgir del desempeño de la labor traductora y del contacto con personas de la cultura meta durante estancias profesionales y personales en un país de la cultura meta.

En el ámbito de la expresión escrita, recomendamos que se enfoque a la producción de textos típicos de la comunicación profesional por un lado (redacción de correos electrónicos, cartas, informes, presentaciones, etc.) y la expresión de experiencias personales por otro. Consideramos que estas últimas, a pesar de no constituir una herramienta de trabajo de un traductor, responden al deseo natural de expresar lo que nos ocurre y fomentan, por lo tanto, la motivación. Nos gustaría destacar que también en la expresión escrita la precisión gramatical y léxica deberían desempeñar un papel importante, puesto que en el mercado laboral se espera de un profesional de los idiomas que sea capaz de expresarse correctamente.

También la enseñanza de las destrezas orales debe orientarse en la comunicación profesional ${ }^{16}$ y personal. Debido a que estos objetivos coinciden con los de la enseñanza comunicativa, la metodología comunicativa es apropiada para su consecución.

\subsection{EL PAPEL DE LA GRAMÁTICA}

Los planteamientos de las diversas metodologías para la Enseñanza de Segundas Lenguas que surgieron durante el siglo $X X$ asignan

\footnotetext{
${ }^{15}$ Según los programas de estudio publicados en Internet.

${ }^{16}$ También Pilar Civera, Ulrike Oster y Amparo Hurtado Albir (1999:72) establecen como uno de los objetivos el desarrollo de la comunicación oral para situaciones de la vida profesional de todo traductor, como por ejemplo, llamadas telefónicas a clientes o asistencia a reuniones de trabajo.
} 
valores muy distintos al estudio de la gramática, que se definen en función de las finalidades de aprendizaje, empezando con el papel destacado que esta ocupa en el método de gramática-traducción hasta llegar a la metodología comunicativa en la que la gramática queda supeditada, en todo momento, a los objetivos comunicativos ${ }^{17}$. La expresión más radical de este planteamiento la encontramos tal vez en el Enfoque por Tareas que selecciona los contenidos gramaticales en función de una tarea comunicativa renunciando, por regla general, a la presentación de sistemas gramaticales completos. Asimismo, el discurso en torno a la enseñanza de la gramática no está exento de polémica, pues el exceso de gramática en las metodologías más tradicionales ha generado un rechazo de la enseñanza de la gramática entre muchos docentes de orientación comunicativa.

Nosotras opinamos que la enseñanza gramatical debe definirse siempre en función de las característas de cada contexto de enseñanza-aprendizaje. La experiencia nos ha mostrado que su efectividad depende, en gran medida, de la formación previa del alumnado y de las estrategias de aprendizaje utilizados por este. En el caso de aprendientes que poseen una buena formación lingüística, que ya han aprendido otros idiomas y que aplican estrategias cognitivas, la ensenanza explícita de sistemas y reglas gramaticales es un instrumento altamente eficaz para conseguir la asimilación del sistema de la lengua meta. En cambio, para los aprendientes que carecen de las características descritas, una enseñanza detallada de la gramática puede resultar abrumadora y desmotivadora.

En el contexto educativo que es objeto de esta contribución, el alumnado comparte, en general, las características arriba descritas (buenos conocimientos lingüísticos, conocimiento de otras lenguas extranjeras, estrategias cognitivas de aprendizaje). Por lo tanto, la enseñanza explícita de la gramática puede favorecer la adquisiciónaprendizaje rápida que la mayoría de los programas de las titulaciones exigen. Esta postura coincide con la de Carmen Gómez García (2002-2003:105), quien postula que

17 Remitimos al lector a los componentes de la Competencia Comunicativa formulados por Michael Canale (1983) y Lyle Bachman (1990). 
la enseñanza de la gramática supone el camino más corto de apropiación de los fenómenos linguísticos de una lengua, el medio más senci. llo de comprensión de textos de elevada dificultad, mas no mediante el análisis de la estructura en sí, no como fin en sí misma, sino como finalidad de comunicarse y ser entendido.

La idea formulada por esta autora de que el aprendizaje de la gramática es un medio y no un fin es ampliamente compartida en el ámbito de la Enseñanza de Segundas Lenguas. Sin embargo, es en la cuestión acerca de en qué medida y cómo usar el medio gramática donde surgen las posturas encontradas.

En la (escasa) bibliografía sobre la enseñanza de lenguas en las carreras de Traducción, encontramos dos posturas divergentes: una que defiende la importancia de su estudio explícito (Carmen Gómez García (2002-2003) y María Ángeles Recio Ariza (2008 y 2011)) y otra enfocada principalmente hacia el trabajo con textos (Laura Berenguer (1999) y Pilar Civera (1996)).

En la descripción de la enseñanza de la gramática en sus clases de Alemán para Traductores, Carmen Gómez García (2002-2003) aboga por la presentación de subsistemas completos de la gramática de la lengua meta y la inclusión de elementos del método de gramáticatraducción con ejercicios enfocados especialmente a la corrección sintáctica y morfológica como el uso de la traducción inversa, relacionando, al mismo tiempo, el estudio de la gramática con el trabajo con textos auténticos incluidos textos literarios en lengua meta. También María Ángeles Recio Ariza (2008 y 2011) subraya la importancia de la gramática en la enseñanza de la Lengua $C$ para Traductores. Según esta autora (2011:168),

la gramática es la base sobre la cual se erige y en la cual se fundamenta la lengua en sí, puesto que presenta el esqueleto sobre el cual se vertebra la lengua.

Para el estudio de la gramática propone un modelo cognitivo que aproveche "los recursos adquiridos en el aprendizaje de otra lengua extranjera o, incluso, de la propia lengua materna" $(2011: 169)^{18}$ y que

${ }^{18} \mathrm{El}$ papel de la lengua materna se tratará en el apartado 4.4. 
reúna las siguientes características: exposición de la lengua extranjera, procesamiento de la misma,

creación de una Gramática Universal y producción en la lengua extranjera. Asimismo, Recio Ariza incide en la importancia de mostrar a los alumnos la logica y sistematicidad que se esconde detrás del sistema gramatical. Los ejercicios que propone comprenden drills no mecánicos, como frases sin sentido que permiten detectar la forma verbal correcta, la construcción de frases a partir de palabras dadas y la corrección de errores cometidos por los alumnos.

La otra postura, aquella que toma como punto de partida el trabajo con textos, se encuentra reflejada en la contribución de Laura Berenguer (1999:136-138), quien dice que

el estudio del sistema de la lengua y de su estructura gramatical debería dejar paso al estudio de otros aspectos más relevantes para la traducción: el estudio de todos aquellos aspectos que el alumno no puede consultar ni en una gramática ni en un diccionario, y que tienen que ver con el discurso y el sentido del texto.

Estos incluyen la dimensión pragmática y la dimensión semiótica. Para este fin, la autora propone un análisis textual basado no solamente en aspectos lingüísticos sino en la Teoría de la Traducción. El modelo que propone incluye factores contextuales, tanto pragmáticos (emisor, receptor, texto), como semióticos (género, connotaciones, referencias y alusiones), intratextuales (coherencia, cohesión y progresión temática) y problemas de traducción.

También Pilar Civera (1996:184) propone el trabajo con textos como punto de partida para el estudio de la gramática:

El estudiante irá descubriendo la gramática a través de los textos, y sus aspectos se amplían o completan al final del estudio del texto; así su planteamiento surgirá de forma progresiva con la dificultad de los textos. Durante la iniciación se proveerá a los estudiantes de las explicaciones mínimas para permitirle la correcta comprensión lectora e iniciarle en la expresión escrita.

Nosotras compartimos con Carmen Gómez García y María Ángeles Recio Ariza la especial atención que merece la instrucción gramati- 
cal en la enseñanza de lenguas a traductores. Para la correcta descodi. ficación de un texto, que constituye, como ya se expuso en apartados anteriores, uno de los objetivos principales de esta enseflanza, los alumnos deben conocer el armazón sintáctico y ser capaces de establecer relaciones entre los diferentes elementos, pues solo a través de la descodificación del sentido denotativo puede el alumno entender cómo funciona el texto en una situación comunicativa. Si bien es verdad que prácticamente la totalidad de la información gramatical necesaria para la comprensión de un texto puede ser recuperada de obras de consulta, cuando el porcentaje de estructuras que deben consultarse es elevado, la comprensión puede verse seriamente mermada. Asimismo, resulta motivador para los alumnos descubrir cómo los conocimientos gramaticales adquiridos les facilitan la construcción de significado de un texto en la lengua extranjera.

En nuestra opinión, el estudio del sistema lingüístico no solo es el camino más rápido hacia el aprendizaje de una lengua extranjera en el contexto que nos ocupa (cf. también Carmen Gómez García: 2002. 2003), sino también un requisito indispensable para un profesional de las lenguas, que necesitará conocer estas no solo como instrumento de comunicación sino también como sistema. Siguiendo estas pautas proponemos un acercamiento a la gramática que reúna las siguientes características:

Comprensiva: se deben presentar subsistemas completos de la lengua meta utilizándolos como marcos generales, cuyos elementos individuales se trabajarán de forma progresiva ${ }^{19}$ (cf. los comentarios de Gómez García 2002-2003: 107)

Cognitiva: se debe insistir en la sistematicidad de los subsistemas gramaticales y fomentar, de esta manera, la creación de una gramática interna de la lengua meta (cf. los comentarios de Recio Ariza 2011: 166-169) mediante la cual el alumno pueda establecer relaciones entre los diferentes elementos que va adquiriendo.

\footnotetext{
${ }^{19}$ Tomando como ejemplo la declinación de los sustantivos alemanes, proponemos presentar ya desde el principio el cuadro completo explicando que los diferentes casos del alemán son formas que aparecen en determinados contextos sintácticos. En el trabajo progresivo de cada uno de los casos y su uso sintáctico se utilizará dicho cuadro como referencia.
} 
Contrastiva: los planteamientos cognitivos de aprendizaje sostienen que integramos lo nuevo en las estructuras cognitivas ya existentes. Desde esta perspectiva no resulta sino coherente y logico incluir los conocimientos de la lengua materna $u$ otros idiomas ya adquiridos en la enseñanza de una lengua nueva. ${ }^{20}$

Pedagógica: por último debe tenerse en cuenta que la capacidad del alumnado para asimilar contenidos gramaticales es limitada. Es, por lo tanto, importante encontrar un camino que compatibilice los objetivos del programa con la capacidad humana de asimilación para evitar la saturación y mantener la motivación. Esto puede conseguirse a través de la inclusión de elementos comunicativos que muestren a los estudiantes que pueden hacer cosas con el idioma y que promuevan un acercamiento lúdico y motivador.

\subsection{EL TRATAMIENTO DE LOS ERRORES}

La investigación en el ámbito de la Adquisición de Segundas Lenguas ha demostrado que los errores forman parte del proceso de aprendizaje de una segunda lengua, pues revelan las hipótesis que los alumnos forman sobre la lengua meta $y$, por lo tanto, las estrategias creativas que están aplicando, y las interferencias que este proceso recibe por parte de la lengua materna, otras lenguas extranjeras o la propia lengua meta. En este contexto surgió también la metodología de investigación del Análisis de Errores, que investiga el origen de los errores que cometen los aprendientes y posibles procedimientos para erradicarlos ${ }^{21}$.

Asimismo, con el surgimiento de la metodología comunicativa cambió la valoración de los errores que empezaron a apreciarse más en función de su impacto comunicativo que en función de su corrección formal, de manera que la corrección gramatical y, en menor medida, la precisión léxica quedaron relegados a un segundo plano.

${ }^{20}$ Este punto se desarrollará con más detalle en el apartado 4.4.

${ }^{21}$ Para un resumen amplio sobre el Análisis de Errores, recomendamos la lectura del capítulo correspondiente en Isabel Santos Gargallo (1993). 
Para el ámbito de la enseñanza de lenguas a traductores, esta valo. ración de los errores es aplicable solo hasta cierto punto, pues la subordinación de la precisión a objetivos comunicativos desemboca en una acentuación de la comprensión global, sin atender a detalles o matices, en las destrezas receptivas y la valoración de los textos producidos en función de su comprensibilidad.

Para las destrezas orales que se han formulado en términos comunicativos, este planteamiento es válido y ayuda, además, a mantener el interés, pues la excesiva corrección en actividades de expresión oral, que ya de por sí son más propensas a generar un mayor número de errores, puede acarrear frustración y rechazo hacia el idioma.

En la expresión escrita, sin embargo, debe insistirse en un correcto uso de la lengua meta. Ya hemos explicado en uno de los apartados precedentes la importancia de una redacción correcta para un traductor aunque no realice traducciones hacia la lengua extranjera. Para fomentar dicha corrección en la expresión escrita, proponemos las siguientes estrategias:

- Extraer ejemplos de errores de la producción de los alumnos y corregirlos en clase en parejas o grupos reducidos $^{22}$. Las frases presentadas deben limitarse a aquellas estructuras que los alumnos ya son capaces de corregir con los conocimientos adquiridos.

- Marcar los errores en las redacciones según una leyenda de símbolos que se reparte el primer día de clase. Los alumnos deben elaborar una segunda versión de su texto a partir de la versión corregida. También aquí recomendamos marcar solo los errores que los alumnos ya deben ser capaces de corregir, dando, si se estima adecuado, pistas acerca de las estrategias a seguir para su corrección. Recomendamos corregir los demás errores directamente, puesto que a pesar de que los alumnos probablemente no estén preparados para asimilar la

\footnotetext{
${ }^{22}$ También María Ángeles Recio Ariza (2008:281) apoya el uso del análisis de errores en la clase de idiomas a traductores.
} 
informacion, no nos parece correcto devolver un texto que todavía contiene errores.

- Llamar la atención de los alumnos sobre fenómenos gramaticales durante el trabajo con textos escritos. Esto aumentará la conciencia lingüística e influirá positivamente en la corrección a la hora de redactar.

\subsection{EL PAPEL DE LA LENGUA MATERNA}

Al igual que la enseñanza de la gramática, también la lengua materna recibe un trato diferente en cada uno de los enfoques didácticos desarrollados, dependiendo, en gran medida, de las teorías de adquisición y aprendizaje que forman la base de cada uno de ellos. A pesar de que en la metodología comunicativa no existen directrices claras sobre su uso, hoy día se tiende más a bien a reducir su uso o prescindir del todo de ella en el aula. En grupos de alumnos de procedencia diversa a menudo no existe alternativa, sin embargo, muchos profesores también aplican esta estrategia en la enseñanza a grupos monolingües atendiendo a directrices por parte del centro, a sus propias creencias acerca de la adquisición de una lengua o simplemente porque esta estrategia goza de mayor prestigio social. ${ }^{23}$

El contexto que nos ocupa aquí, sin embargo, se caracteriza de otros contextos educativos precisamente por el papel de la lengua materna, pues la finalidad de la enseñanza consiste en preparar a los estudiantes a una actividad que implica el trabajo constante con dos códigos lingüísticos. No resulta, por lo tanto, sino coherente integrar un enfoque contrastivo en la enseñanza de lenguas.

Esta postura también es compartida por muchas de las autoras citadas. Laura Berenguer (1999:139) defiende un enfoque contrastivo tanto a nivel lingüístico como textual, Pilar Civera (1996:184) propone analizar, de forma contrastiva, "las dificultades más comunes y ele-

${ }^{23}$ Es una creencia popular muy extendida en España de que una enseñanza que se realiza únicamente en la lengua meta es más efectiva que una enseñanza con apoyo de la lengua materna. 
mentos de discrepancia" entre las dos lenguas, Carmen Gómez García (2002-2003:105) destaca que el aprendiente adulto imbrica lo nuevo en redes asociativas y María Ángeles Recio Ariza (2011:169) recuerda la importancia de aprovechar los recursos ya adquiridos durante la adquisición de otros idiomas, incluyendo la lengua materna.

En nuestra opinión, tal como proponen también varias de las autoras citadas, la enseñanza de lenguas debe formularse desde los planteamientos del aprendizaje cognitivo que sugieren que la asimilación de información nueva se efectúa mediante la comparación con los conocimientos ya adquiridos. En consecuencia, los mapas mentales ya existentes condicionan la representación e integración de lo nuevo, que, a su vez, puede retroalimentar y modificar dichos mapas. Aplicado al ámbito de la adquisición-aprendizaje de idiomas, esto significa que, al menos durante las primeras etapas, dicha comparación e integración se efectúa desde las estructuras de la lengua materna o de otra lengua extranjera según las similitudes y diferencias, algo que se manifiesta en la preocupación por parte de los alumnos por disponer de una traducción o en la búsqueda de similitudes con la lengua materna u otra lengua estudiada (normalmente el inglés) y el consiguiente alivio si dicha analogía existe.

Debido a nuestra actividad docente y la experiencia propia como aprendientes de varios idiomas extranjeros, estamos convencidas de que la lengua materna desempeña un papel importante en el proceso de aprendizaje de un idioma extranjero en el aprendiente adulto. En este contexto, los planteamientos básicos hechos por el Análisis Contrastivo nos parecen, en principio, acertados:

We assume that the student who comes in contact with a foreign language will find some features of it quite easy and others extremely difficult. Those elements that are similar to his native language will be simple for him, and those elements that are different will be difficult. (Lado 1957:2)

En los años 60, estas premisas se llevaron a la didáctica mediante un enfoque conductista y fueron desechadas junto a este debido a que dicho enfoque resultó ineficaz y poco motivador. Asimismo, la hipótesis formulada por el Análisis Contrastivo de que la dificultad se 
traduciría en un número significativo de errores nunca se vio confirmada por estudios empíricos.

No obstante, poco se ha explorado el potencial de estos planteamientos como estrategia de aprendizaje debido a la mala fama que adquirió el Análisis Contrastivo por su fracaso como instrumento de pronóstico y la creencia muy extendida entre docentes de idiomas de que se debe desterrar la lengua materna de los aprendientes de las clases.

Siguiendo los principios de aprendizaje cognitivos arriba propuestos, nosotras, sin embargo, creemos que el empleo de la lengua materna como elemento contrastivo puede resultar en una enseñanzaaprendizaje más eficaz en el contexto que describimos, pues a través de la contrastación pueden detectarse potenciales áreas de dificul$\operatorname{tad}^{24}$ La enseñanza contrastiva puede abarcar los siguientes ámbi$\operatorname{tos}^{25}$ :

- La gramática: partimos de la premisa de que un (futuro) traductor debe conocer las estructuras de las lenguas extranjeras con las que trabaja y la de la suya propia y, además, la terminología básica para describir y analizar fenómenos gramaticales. A través de la enseñanzaaprendizaje contrastiva, los alumnos conocerán los subsistemas de la lengua meta y los analizarán desde el subsistema correspondiente de su lengua materna. Esta comparación ayudará a los aprendientes a tomar

24 Nos gustaría reiterar que las propuestas formuladas no son necesariamente transferibles a otros contextos educativos, por ejemplo, a grupos con alumnos de diversa procedencia o a alumnos que no poseen conocimientos metalingüísticos. ${ }^{25}$ Cf. también los comentarios de Carmen Valero Garcés (1998-99: 33-34), quien destaca los beneficios de la enseñanza contrastiva a través de textos:

Working with parallel texts may also be used to illustrate specific aspects of the structure of the language (passive voice, prepositions or conditional sentences), or aspects connected with vocabulary and context (rhetoric conventions) or pragmatic considerations (cultural differences, ways of expressing politeness, etc.), because through the inevitable comparison of both languages, the student may easily assimilate the structural, semantic and pragmatic differences between the languages and becomes more aware of them. 
conciencia no solo de las diferencias estructurales entre ambos idiomas, sino también a adoptar una actitud reflexiva hacia de su propia lengua. De esta manera, los estudiantes descubrirán que los idiomas poseen diferentes mecanismos de expresión y que cada uno comprende fenómenos que no encuentran correspondencia en otro.

- Como estrategias para el aula proponemos la elaboración de cuadros por parte de los alumnos a partir de material lingüístico (semi)auténtico, la descripción de las diferencias halladas y la traducción inversa.

- El léxico: también en el ámbito léxico, el trabajo contrastivo puede ayudar a aclarar significados y prevenir asociaciones equivocadas que pueden darse en la enseñanza monolingüe, pues especialmente en las primeras etapas, las definiciones en lengua meta resultan demasiado complicadas o simplistas y el alcance de las imágenes, de los dibujos y de la mímica es limitada más allá de los objetos y las acciones concretos. La traducción puede suplir estas deficiencias y asegurar que los alumnos no se formen ideas equivocadas. ${ }^{26}$ Mientras en etapas tempranas, la lengua materna puede usarse principalmente para controlar la comprension y comparar redes semánticas entre los dos idiomas, en fases posteriores puede emplearse para llamar la atención de los alumnos sobre las diferentes asociaciones que palabras aparentemente equivalentes provocan en los hablantes recordando a los aprendientes que los idiomas están

\footnotetext{
${ }^{26}$ Respecto a este punto, recuerdo la siguiente anécdota: un alumno que me había solicitado ayuda para superar un examen de alemán de nivel A1 me dijo en una ocasión que dabei significaba cartera. Cuando le pregunté cómo había llegado a esta asociación me dijo que su profesora, cuando le preguntó por el significado, había golpeado su torso a la altura del pecho queriendo indicar que llevaba la cartera encima. El alumno, sin embargo, había interpretado que se refería a la cartera en sí.
} 
ligados a conceptos y valores culturales que encuentran expresion a través de ellos. ${ }^{27}$

- Aspectos pragmáticos: por último queremos destacar que la enseñanza contrastiva no debe pararse en el nivel de la estructura o de la palabra. El análisis contrastivo de textos tal como lo plantea Laura Berenguer (1999) no solo ayudará a los aprendientes a tomar conciencia sobre las convenciones culturales de organización textual sino también del empleo de las diferentes estructuras a nivel microtextual y su funcionamiento en un contexto auténtico.

Vamos a cerrar este apartado con unas reflexiones acerca de la lengua en la que debe realizarse la instrucción. Sería deseable que la mayor parte de la comunicación en el aula se efectuara en la lengua meta para que la exposición del alumno a esta sea máxima. Sin embargo, en el caso de gran distancia interlingüística entre la lengua materna y la lengua meta, este enfoque a menudo resulta ineficaz, especialmente en etapas tempranas, puesto que las destrezas orales en lengua meta todavía no están desarrolladas y la aplicación de estrategias cognitivas precisa de un lenguaje que el alumno no posee todavía. Proponemos, por lo tanto, un acercamiento paulatino al uso de la lengua meta como instrumento de comunicación en el aula en función de la mutua inteligibilidad de las dos lenguas.

\subsection{EL USO DE LA TRADUCCIÓN}

Al igual que la enseñanza de la gramática, también el uso de la traducción en la clase de idiomas ha dado lugar a posiciones encontradas. La postura de los detractores queda bien resumida en la advertencia de Isabel Santos Gargallo (1993:61) de que la traducción en clase de idiomas se puede convertir en un hábito difícil de erradicar y tener una influencia negativa sobre la construcción sintáctica en la lengua meta. Según esta autora, puede constituir una barrera para el alumno

${ }^{27}$ Como ejemplo, invitamos al lector a reflexionar sobre las diferentes asociaciones culturales y políticas que provocan las palabras Heimat y patria.

Futhark 9 (2014)

Schmidhofer, La especificidad, 425-452

ISSN $1886-9300$ 
a pensar en la lengua meta. Entre los defensores, citaremos a Henry Widdowson (1978, citado en Carmen Valero Garcés 1998-99:31), quien sostiene que el objetivo de la enseñanza reside en que el alumno perciba la lengua meta como instrumento de comunicación igual que su lengua materna, lo que justificaría el uso de la traducción.

Los argumentos esgrimidos en contra de su uso en la enseñanza con fines comunicativos, que advierten del peligro de la asociación de elementos de la lengua meta con otros de la lengua materna, no son aplicables al contexto que nos ocupa, pues se prepara al alumnado a trabajar con dos códigos lingüísticos $\mathrm{y}$, precisamente, a traducir. La inclusión de la traducción en las clases también es compartida por las siguientes autoras que se han ocupado de la especificidad de la enseñanza de lenguas en las carreras de Traducción: Carmen Gómez García, (2002-2003:109), María del Carmen Martín Santana (2004), María Ángeles Recio Ariza (2008: 281, basándose en Gerhard Pisek (1994)) y Amparo Hurtado Albir (1987, citada en Isabel Santos Gargallo 1993: 61-62). Los argumentos que más relevantes nos parecen pueden resumirse de la siguiente manera:

El uso de la traducción...

- fomenta la capacidad de análisis y síntesis

- permite relacionar la lengua materna con la lengua extranjera $y$ tomar conciencia de las diferencias estructurales, léxicas y sociolingüísticas

- es el punto de partida de debates sobre fenómenos lingüísticos

- permite la práctica de fenómenos ya estudiados

- fomenta la precisión en procesos de comprensión

- sirve para la ampliación del léxico

- mejora la expresión y la flexibilidad de expresión en la lengua materna

- permite tomar conciencia de las interferencias en diferentes niveles 
Para la aplicación en el aula recomendamos, tal como expusimos en el apartado 4.1, el uso de traducciones pedagógicas enfocadas al análisis de los textos originales y el trabajo contrastivo entre la lengua extranjera y la lengua materna.

\section{CONCLUSIÓN}

El análisis de objetivos y motivaciones presentado al inicio de este artículo ha mostrado que la enseñanza de idiomas en las carreras de Traducción precisa de una metodología específica que dé respuesta a estas necesidades. Partiendo de la metodología más extendida hoy día, la comunicativa, hemos intentado esbozar las bases de una enseñanza diseñada a preparar al alumno universitario para el desempeño de la traducción. Creemos que esta área, que se nutre tanto de la Didáctica de Segundas Lenguas y como de la Traductología, ha recibido poca atención debido a su carácter híbrido y que merecería un debate más intenso acerca de sus principios metodológicos y aplicaciones prácticas, pues la experiencia nos ha mostrado que especialmente en las asignaturas de traducción desde la Lengua $C$, en la que los alumnos no suelen tener conocimientos previos al iniciar los estudios, existen, a menudo, deficiencias lingüísticas que podrían evitarse con una enseñanza de idiomas más enfocada a las necesidades de los futuros traductores.

\section{BIBLIOGRAFÍA}

BERENGUER, Laura (1996): “Didáctica de segundas lenguas en los estudios de traducción", en: Hurtado Albir (ed.): La enseñanza de la traducción, Castellón,

Publicacions de la Universitat Jaume I, 9-29.

- (1999): "Cómo preparar la traducción en la clase de lenguas extranjeras", en: Quaderns, Revista de traducció 4, 135-150.

BREHM CRIPPS, Justine y Hurtado Albir (1999): “La primera lengua extranjera”, en: Hurtado Albir, Amparo (dir.): Enseñar a traducir, Madrid, Edelsa, Colección investigación didáctica, 59-70.

CIVERA GARCíA, Pilar (1996): “La enseñanza de la lengua C", en: Hurtado Albir 
(ed.): La enseinanza de la traducción, Castellón, Publicacions de la Universitat Jaume I, 183-188.

GNera García, Pilar, Oster Ulrike y Amparo Hurtado Albir (1999): "La segunda lengun extranjera", en: Hurtado Albir, Amparo (dir.): Enseñar a traducir, Madrid, Edelsa, Colección investigación didáctica, 71-86.

GOMEz GaRCia, Carmen (2002-2003): "Sobre la necesidad de contravenir

las reglas de las mueoas metodologias", Encuentro Revista de investigación e innovación en la clase de idiomas 13-14, 101-112.

JAMrES, Carl (1980): Contrastioc Analysis, London etc, Longman.

KuSsMAur, Paul (1995): Training the translator, Amsterdam/Philadelphia, John

Benjamins.

LADO, Robert (1957): Linguistics across cultures, Michigan, Ann Arbor.

MARTín SANTANA, María del Carmen (2004): "La importancia de la lengua C en la facultad de Traducción e Interpretación: un concepto de aprendizaje de la lengua con un fin especifico", en: Bravo Utrera (ed): Traducción, lenguas, literaturas: sociedad del conocimiento, enfoques desde y hacia la cultura, Las Palmas de Gran Canaria, Universidad de Las Palmas de Gran Canaria, Servicio de Publicaciones, 141-152.

Pérez LaCARTA, Ana María (2009): "Comprender para traducir", Thélème.

Revista Complutense de Estudios Franceses, 24, 163-170.

RECIO ARIZA, María Ángeles (2008): “Das Warum und das Wie des

Sprachunterrichts (Deutsch) an den Übersetzungs- und Dolmetscherfakultäten

Spaniens", en: Pichler, Georg (coord.): Germanística y enseñanza del alemán en

España, Madrid, editorial idiomas, 277-284.

- (2011): “La Gramática Cognitioa y su utilidad en la enseñanza de la

lengua extranjera para Traductores/Interprétes", en: RoIss, Silvia et alii (eds.): En

las vertientes de la traducción e interpretación del/al alemán, Berlin, Frank\&Timme, 163-173.

SANTOS GARGallo (1993): Análisis Contrastivo, Análisis de Errores e Interlengua en el marco de la Lingüística Contrastiva, Madrid, Síntesis.

SCHMIDHOFER, Astrid (2012): "La comprensión lectora en el proceso de traducción. Análisis de los errores de comprensión en la traducción de textos

periodísticos alemanes por parte de hispanohablantes", Skopos. Revista

Internacional de Traducción e Interpretación, 1, 65-87. ISSN: 2255-3703.

VALERO GARCÉs, Carmen (1998-99): "Some pedagogical and practical implications of contrastive studies in ELT", RESLA 13, 27-36. 